\title{
Trivium
}

Revue franco-allemande de sciences humaines et sociales - Deutsch-französische Zeitschrift für Geistesund Sozialwissenschaften

11 | 2012

Gustave Flaubert. A l'Orient du réalisme

\section{Jehova gleich Moloch: Eine »antireligiöse« Lesart von Salammbô}

\section{Agnès Bouvier}

Traducteur : Erika Mursa

\section{CpenEdition}

Journals

Édition électronique

URL : http://journals.openedition.org/trivium/4270

DOI : $10.4000 /$ trivium. 4270

ISSN : 1963-1820

Éditeur

Les éditions de la Maison des sciences de l'Homme

Référence électronique

Agnès Bouvier, « Jehova gleich Moloch: Eine »antireligiöse« Lesart von Salammbô », Trivium [Online],

11 | 2012, online erschienen am 29 Juni 2012, abgerufen am 08 September 2020. URL : http://

journals.openedition.org/trivium/4270; DOI : https://doi.org/10.4000/trivium.4270

Ce document a été généré automatiquement le 8 septembre 2020.

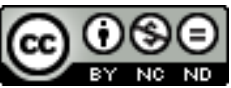

Les contenus des la revue Trivium sont mis à disposition selon les termes de la Licence Creative Commons Attribution - Pas d'Utilisation Commerciale - Pas de Modification 4.0 International. 


\title{
Jehova gleich Moloch: Eine "antireligiöse« Lesart von Salammbô
}

\author{
Agnès Bouvier \\ Traduction : Erika Mursa
}

\section{NOTE DE L'ÉDITEUR}

Wir danken Agnès Bouvier, der Zeitschrift Romantisme sowie dem Verlag Armand Colin für die freundliche Genehmigung, diesen Artikel zu übersetzen.

1 Wie ist die »antireligiöse Wut« zu verstehen, von der Flaubert während der Schaffensjahre an Salammbô beherrscht wurde? Ist die Radikalisierung seiner Haltung dem Zufall zu verdanken oder gibt sie Auskunft zur Interpretation des Romans? Oder vielmehr: Was hat Flaubert während seiner Recherchen zu Karthago entdeckt, das ihn so sehr gegen das Christentum aufbrachte, dass sich sogar manche seiner Briefpartner darüber ereiferten? ${ }^{1}$

Als Ausgangspunkt wählen wir das Datum des 27. Oktober 1859. Frédéric Baudry bringt Flaubert wegen einer Detailfrage zum Peplos der Astarté ${ }^{2}$ mit Renan in Kontakt und verschafft ihm damit Zutritt zu einem Kreis, in dem die Beschäftigung mit Karthago und Phönizien einhergeht mit einem gemeinsamen »Hass auf die Priester «, denn die um Renan versammelten Gelehrten zeichnen sich gleichermaßen durch ihre Kenntnisse der phönizischen Welt wie ihren Antiklerikalismus aus. Dies gilt beispielsweise für Alfred Maury, der zu einem Berater Flauberts in religiösen Fragen wird und der sich durch seine leidenschaftlichen Positionen wider eine biblische Interpretation der Ursprünge der Menschheit hervortut. ${ }^{3}$ Die "Dîners« der Jahre 18591860 dürften in dieser Hinsicht für Flaubert von erheblicher Bedeutung gewesen sein, und der Boulevard du Temple wurde zu einem der zentralen Stätten des gelehrten Antiklerikalismus: "An eben diesem Sonntag gab es bei mir eine große Auseinandersetzung zwischen Baudry, Saint-Victor und dem hervorragenden Vater Maury, der charmant ist. Ich werde morgen mit ihm und Renan in Versailles dinieren.« 
${ }^{4}$ Die fragliche Auseinandersetzung dreht sich um das Examen critique des doctrines de la religion chrétienne (Kritische Untersuchung der Lehren der christlichen Religion) von Patrice Larroque, ein am 27. April 1860 auf den Index gesetztes Werk, in dem der Autor durch eine historische Bibelexegese zu belegen sucht, dass die grundlegenden Dogmen des Christentums nicht akzeptabel sind. Das Buch, das Flaubert nach seiner Rückkehr nach Croisset verschlingt, macht starken Eindruck auf ihn. Die Lektüre von Larroque und Michelet ist in der Zusammenfassung der Woche vom 8. Mai 1860, die er Ernest Feydau gegenüber macht, zwischen zwei Anmerkungen zu Salammbô erwähnt:

»Endlich überarbeite ich mein nicht enden wollendes Kapitel VII, das mir einige Mühe kostet. Außerdem gehe ich an die Vorbereitung des VIII. Kapitels. Ich stopfe mich voll mit den antikatholischen Argumenten des Vaters Larroque, ich lese den letzten Band von Michelet und verschiedene Artikel zur Bibelauslegung in der Revue germanique. Seit meiner Rückkehr habe ich 15 Seiten geschrieben, was für einen Verbissenen wie mich ganz ansehnlich ist. ${ }^{5}$

Die gleichzeitige Präsenz von "punischem « Schreiben und "antikatholischer« Lektüre ist mehr als ein Zufall: Die Welt Karthagos und die der Bibel haben durchaus etwas miteinander zu tun, Flaubert weiß dies seit seiner ersten Recherche-Arbeit für Salammbô, in deren Verlauf er die Bibel von Samuel Cahen gelesen und mit Anmerkungen versehen hat. ${ }^{6}$ Renan und seine Kollegen von der Académie des Inscriptions konnten ihn nur darin bestätigen, dass die Gleichsetzung karthagisch = phönizisch = hebräisch, auf der ein großer Teil seiner »Herleitungen « gründet, zutrifft. Im Brief vom 27. Oktober 1859 ging es genau um eine Passage aus jenen Neuen Betrachtungen über den allgemeinen Charakter der semitischen Völker und insbesondere über ihren Hang zum Monotheismus, die im Zusammenhang mit dem Peplos der Astarté insofern interessant ist, als Renan in dem, was zum "Zaimph« werden sollte, einen "Stoff bedeckt von Schriftzeichen" sieht, dessen Status dem der »Thorah (Gesetzbuch) der Hebräer « ${ }^{7}$ nahe kommt. Renan gründete seine Beweisführung auf die lautliche Ähnlichkeit des phönizischen Thouro und des hebräischen Thorah. In allen seinen Werken weist er unablässig auf die Analogien zwischen beiden Sprachen hin: »Es dürfte selbst bei fehlenden schriftlichen Zeugnissen keinerlei Zweifel am Charakter der phönizischen Sprache und ihren Analogien zum Hebräischen verbleiben « $^{8}$; und er schreibt sogar: »Die Inschrift von Marseille ist entweder wegen ihres Alters oder wegen ihrer karthagischen Herkunft fast reines Hebräisch «. ${ }^{9}$ Das Punische hat daher gemäß Renan mehr als eine verwandtschaftliche Beziehung zum Hebräischen, denn Karthago war tatsächlich ein Ort, an dem die hebräische Sprache bewahrt wurde gemäß jenem Gesetz, wonach "man nicht selten beobachten kann, dass eine Kolonie die Sprache unverfälschter konserviert als das Mutterland «. ${ }^{10}$

Unter diesen Umständen und da Renan eine notwendige Verbindung zwischen Sprache und Religion als zwei miteinander einhergehende, spontane Hervorbringungen der "Rasse" sieht, innerhalb der sie entstehen, erstaunt es nicht, dass eine Nähe zwischen hebräischem und phönizischem Kult und eine Verwandtschaft zwischen deren heiligen Texten gesehen wird:

»Wenn man die verschiedenen semitischen Kosmogonien, die uns überliefert sind, miteinander vergleicht, jene aus dem ersten Kapitel der Genesis, jene von Berossos, von Sanchuniathon oder von Mochus, jene, die uns von Eusebius und Damaskios erhalten sind, ist man schon auf den ersten Blick von deren Ähnlichkeit überrascht. «11

Das Problem, das sich daraufhin stellt, ist die Frage nach der Verbindung zwischen Religionen, die in ihrer Vorstellung von der Gottheit nicht nur unterschiedlich, 
sondern offenkundig auch antagonistisch sind. Die Antwort ist von kapitaler Bedeutung, da sie den historischen Status der jüdischen Religion und somit auch der christlichen Religion betrifft: Ist deren Aufkommen im Laufe der Geschichte eine Ausnahme oder nicht? Renan entscheidet sich für eine Lösung, mit der die jüdische Ausnahme zugleich aufgehoben und bestätigt wird, indem er sie in den von ihm so bezeichneten Semitismus einfügt. Um seine These zu stützen, wonach sich das Genie der "semitischen Rasse« durch intuitiven Monotheismus definiert, will er zeigen, dass die phönizische Religion unter ihren polytheistischen Manifestationen tatsächlich zutiefst monotheistisch ist und dass die verschiedenen Götter Baal, Moloch, El etc. nur Variationen ein und derselben Idee einer Gottheit und nicht verschiedene Entitäten sind, da jeder göttliche Name nur Synonym oder Beiname für die anderen ist. »Moloch" wäre somit als eine Bezeichnung für Jehova zu sehen, was schon sehr beunruhigend ist.

Es könnte einem aber auch leicht die gegenteilige Hypothese in den Sinn kommen: Anstatt in der phönizischen Religion einen verbrämten Monotheismus zu sehen, kann man die hebräische Religion als einen verdeckten grundlegenden Polytheismus in Form eines späten Monotheismus sehen. In diesem Fall würde sich die Gleichsetzung "Moloch ist gleich Jehova" umkehren in "Jehova ist gleich Moloch«. Dies ist eine ungeheuerliche Idee, die jedoch im 19. Jahrhundert kursierte und auch Flaubert bekannt war; sie wurde in einem Werk formuliert, das in der Bibliographie enthalten ist, die der Autor von Salammbô 1862 bei der Antwort auf die Vorwürfe von Guillaume Froehner zusammenstellt. ${ }^{12}$ Es handelt sich dabei um ein eigenartiges Buch, das auf Kosten des Autors 1850 bei Ladrange veröffentlicht wurde und auszugsweise Übersetzungen und Zusammenfassungen deutscher Werke enthält unter dem Titel: Qu'est-ce que la Bible, d'après la nouvelle philosophie allemande? (Was ist die Bibel, gemäß der neuen deutschen Philosophie?). ${ }^{13}$ Der Übersetzer und Kommentator Hermann Ewerbeck will die neuhegelianische Philosophie in Frankreich durch eine Streitschrift verbreiten, die als Waffe gegen die stets bedrohliche »Infame« (l'inf.) gedacht ist..$^{14}$ In diesem Sinne setzt er ihr die radikalsten Schriften der um Feuerbach versammelten deutschen Atheisten entgegen. Der erste übersetzte Beitrag Daumers mit dem Titel »Le culte du Moloch chez les Hébreux de l'antiquité« (= Daumer [1842]) beginnt mit folgendem Satz: »1. Jehova und Moloch waren am Anfang ein und derselbe Gott.« ${ }^{15}$ Und der Text fährt folgendermaßen fort:

»2. Der Moloch-Kult wurde unter den Hebräern nicht vom großen König Salomo begründet; dieser Kult war im Gegenteil deren nationale und ursprüngliche Religion seit Abrahams Zeiten und ihm wurde unter Abraham, Moses, Samuel, David bis hin zu Salomo gehuldigt.

3. Das Opfer von Menschenfleisch, das in den Flammen des Altars oder auf andere Weise dargebracht wurde, war sowohl dem Molochismus als auch dem Jehovismus wesentlich. Die biblische Geschichte und die Schrift der Hebräer erklären mit großer Offenheit, dass diese Art der Verehrung des nationalen Gottes nicht nur erlaubt, sondern unter Moses, unter den Richtern und unter König David streng angeordnet war.

4. Die Hebräer der Antike töteten ihre Feinde und Fremde vor dem Altar oder warfen sie bei lebendigem Leib in die Flammen dieses Jehova-Moloch-Altars; ebenfalls Angehörige ihrer eigenen Nation und - wohlgemerkt - nicht nur Verbrecher, sondern auch unschuldige Menschen, zuweilen sogar ihre Kinder, ihre Herrscher und hochrangigen Priester.

5. Die ursprüngliche Religion Moses befahl, bevor sie abgemildert wurde, zu Ehren von Moloch-Jehova ohne Ausnahme die männlichen Erstgeborenen unter den Tieren und erst recht unter den Menschen zu töten. 
6. Die Opferung von lebendigen Kindern, die in die feuerglühenden Arme der Bronzestatue geworfen wurden, diese unsägliche Grausamkeit, die nur durch einen pathologischen menschlichen Geist zu erklären ist, wurde bereits von Abraham auf dem Berg Moria und von David auf der Tenne Aravnas praktiziert; hier ließ Salomo später von phönizischen Baumeistern den Tempel der Stadt Jerusalem errichten. Der berühmte Bronzealtar des Mosestempels ebenso wie des Salomotempels war nichts anderes als jene bronzene Götzenstatue, die dazu diente, die dem JehovaMoloch geweihten Kinder bei lebendigem Leib $\mathrm{zu}$ verbrennen und in Asche aufgehen zu lassen, gemäß hebräischem oder phönizischem oder kanaanäischem Kult; da diese drei Stämme zur gleichen Familie gehörten, hatten sie einen gemeinsamen Kult.«

Es sei daran erinnert, dass diese Zeilen aus einem Buch stammen, das Flaubert in seine Bibliographie zu Salammbô aufnimmt, wobei das nachträglich erstellte Dokument nicht in seiner Vollständigkeit veröffentlicht werden, sondern als Grundlage für eine rückblickende, polemische Reflexion über die Quellen des Romans dienen sollte. Das Echo, das diese Seite beim Leser von Salammbô hervorruft, und das in mehr als einer Hinsicht erhellende Licht, das damit unvermittelt auf das ideologische Umfeld des Werkes gerichtet wird, werfen die Frage auf, inwiefern der Romancier dabei mit wissenschaftlichem Bewusstsein vorgegangen ist. Sollte Flaubert Daumers Ausführungen lediglich die »bildgewaltigen« Elemente entnommen haben, ohne die dazugehörige These zu berücksichtigen? Gewiss nicht.

Die Gleichsetzung »Jehova ist gleich Moloch« erscheint ausdrücklich im Dossier zur Bibel von Cahen, das Flaubert schon im Juli 1857 erstellt:

»Jehova = Moloch Jehova. s sein Zorn lodert, sein Feuer ist gewaltig, seine Lippen sind voll wütendem Geschrei und seine Zunge ist ein verschlingendes Feuer «. 30,27« »Jehovah = Moloch Jehovah. ısa colère brule, son feu est violent, ses lèvres sont pleines de fureur \& sa langue un feu devorant«. 30,27 (fo 313; Jesaja, Band IX, XXX, 27, S. 112)《

Die Annäherung zwischen Jehova und Moloch, zweier Sonnengötter, zweier verschlingenden Götter, ist hier reiner Flaubert: Nichts dergleichen findet man in der entsprechenden Anmerkung von Cahen. Hier handelt es sich um eine kommentierte Exegese, die der Romancier auf eigene Faust vornimmt. Flaubert formuliert willentlich die Gleichsetzung von hebräischem Gott und phönizischem Gott; es handelt sich nicht um eine unbewusste Assoziation von Ideen, sondern um eine Aussage, die mit dem offenkundigen Zeichen von Wissenschaftlichkeit vorgebracht wird, wovon Salammbô lange gezeichnet bleiben sollte.

Dank der von Geneviève Mondon ${ }^{16}$ entschlüsselten handschriftlichen Skizzen kann man die Entstehung jener Passage nachvollziehen, in der Schahabarim sich die Frage nach der Verwandtschaft zwischen hebräischer und kanaanäischer Religion stellt. Die Handlungsskizze (fo 104) beschließt die religiösen Überlegungen Schahabarims mit folgenden Worten: »neigte zum Monotheismus der Hebräer, zweifelte an der geltenden Kosmogonie«. Das Thema wird folgendermaßen entwickelt:

»A) >Verbindung zu Hebräern \& Griechen. er empfing sie heimlich des Nachts bei sich / \& dieser einzige Gott verwirrte ihn / \& er sah, fand so viele Beziehungen zu Moloch, dass er den Unterschied nicht sah.، (fo $75 \mathrm{Vo}$ )《

»B) >Der absolute Gott, den die Meister des Gesetzes ihm in Jerusalem erklärt hatten, quälte ihn` (fo $136 \mathrm{Vo}$ )«

»A) rrelation avec les Hebreux \& les Grecs, il en recevait chez lui la nuit, secretement / \& ce dieu unique le troublait / \& voyait trouvait tant les rapports avec Moloch qu'il n'en voyait la différence.، (fo $75 \mathrm{Vo}$ )« 
»B) \Le Dieu absolu que les maîtres de la Loi lui avaient expliqué à Jerusalem, le tourmentait< (fo $136 \mathrm{Vo}$ ) «

Diese interne theologische Debatte Schahabarims über »diesen einzigen Gott«, der Moloch so ähnlich war, »dass er den Unterschied nicht sah«, wird in der endgültigen Version unterdrückt. Dennoch: die Schockwelle des »Jehova ist gleich Moloch« hat eine Verwirrung hervorgerufen (»dieser einzige Gott verwirrte ihn«), die ihre Wirkung auf die schließlich abtrünnige und ratlose Person Schahabarims haben sollte.

Die Verstörung, die diese Kontamination zwischen den Göttern hervorrief, setzt sich bis ins christliche Zeitalter fort. Wenn Daumer mit so viel Nachdruck den Molochismus der Hebräer unterstreicht, dann weniger aus Antisemitismus ${ }^{17}$ denn aus Antichristianismus. So heißt der zweite Teil seiner Untersuchung, die 1847 veröffentlicht und auf Seiten 49-173 des Bandes von Ewerbeck wiedergegeben wurde, "Secrets de l'antiquité chrétienne " [Die Geheimnisse des christlichen Alterthums]; darin soll das Wesen des Christentums in einem "Molochismus« gefunden werden, der die Reform des "moralischen Jehovismus", den die Juden nach der Gefangenschaft einführten, überlebte. Wenn man es also richtig versteht, so ist der Molochkult aus der Religion der Hebräer nach der Rückkehr aus Babylon verschwunden. Vollständig? Nein, eine kleine jüdische Sekte bewahrt noch immer die alten Riten, was folgenlos geblieben wäre, wenn sich die betreffende Sekte nicht letztendlich auf der ganzen Welt durchgesetzt hätte - nämlich die Christen. Das Christentum bedeutete demnach die Verstetigung des molochistischen Menschenopfers und die Kommunion ein kannibalisches Ritual, das die ersten Christen ganz unmetaphorisch praktizierten. Und selbst nach der zumindest offiziellen Abschaffung dieser Praktiken bleibt die christliche Faszination für das verschlingende Feuer erhalten. Das Christentum errichtet die Herrschaft von Scheiterhaufen, Enthaltsamkeit und Kasteiung, von Verstümmelungen, Folter und Wahn, mit einem Wort: von Anti-Natur.

»Aus diesem fürchterlichen Princip der Negation und Abstraktion fließen alle Fanatismen und Gräuel, die die Geschichte des Christenthums beflecken, und diese sind keineswegs etwas dem Wesen dieser Religion Fremdes, nicht ganz nur aus ihrem eigensten, innersten Grunde Hervorgehendes, von ihr urtheilend Abzutrennendes, sondern ihre wahre, charakteristische, nothwendige und unvermeidliche Entwicklung und Manifestation. ${ }^{18}$

Der Einfluss von Daumers Ideen auf Feuerbach ist offensichtlich: Der Autor von Das Wesen des Christentums bezieht sich mehrfach auf ihn ${ }^{19}$ und manche Passagen seiner berühmten Abhandlung sind direkt aus den Geheimnissen des christlichen Alterthums hervorgegangen. Ewerbeck unterstreicht »die seltsame Unbekümmertheit, mit der die Kirche den menschlichen Körper tötet ${ }^{20}$ und fügt als Anmerkung hinzu:

"Die religiösen Massaker an den Häretikern haben den Molochkult ersetzt. Dies lässt sich mühelos durch kritischen Verstand und Logik beweisen; ich will hier nur einen historischen Beweis anführen: in der Nähe von Sevilla Lorente existiert noch nach 1800 das Quemadero, ein steinernes Schafott, das vor drei Jahrhunderten vom Großinquisitor in der Ebene von Tablada errichtet wurde, mit vier kolossalen Gipsstatuen (im Volk die vier Propheten genannt), an denen das zu verbrennende Opfer festgebunden wurde; manche sagen, dass es in ihrem Innern verbrannt wurde. ${ }^{21}$

»Das »Quemadero« der Inquisition wäre demnach lediglich eine Abart der Bronzestatue des gefräßigen Moloch: »Herr! Verzehre sie! ««22

Daumers Ideen, so überzogen sie auch erscheinen mögen, haben in Deutschland eine keineswegs marginale Anhängerschaft gefunden. Wenn man an die Wirkung des Feuerbachschen Werkes denkt, in dem diese Ideen explizit aufgegriffen wurden und 
dessen Einfluss dauerhaft bei einer ganzen Generation von Philosophen wahrnehmbar war, lassen sich die Folgen der Theorie vom hebräischen Molochismus auf das deutsche Denken ermessen. In Frankreich ist die Rezeption, selbst in den am stärksten "antireligiösen« Kreisen, offensichtlich besonnener (von den anderen, die beiderseits des Rheins Skandal schreien und von Wahn sprechen, ist hier natürlich nicht die Rede). In der Juni/November-Ausgabe 1850 von La liberté de penser ${ }^{23}$ veröffentlicht Renan eine sehr negative Kritik der beiden von Ewerbeck veröffentlichten Bände. Der Artikel verurteilt vor allem die Heftigkeit, mit der in Das Wesen des Christentums der Atheismus zum Ausdruck kommt. Er kritisiert auch die im zweiten Band übersetzten Autoren, ohne sie namentlich zu nennen: „Es ist ärgerlich, dass der Übersetzer, dessen Uneigennützigkeit lobenswert ist, jene Schriften, die man zur Kenntnis nehmen sollte, mit völlig wertlosen Stücken vermischt hat, von denen manche in keiner Weise ernst genommen werden können.« Was nicht ernst genommen werden kann, betrifft sehr wahrscheinlich die Arbeiten von Daumer. Dieselben Vorbehalte bei Patrice Larroque, und fast in gleicher Formulierung: Über das Buch von Lützelberger mit dem übersetzten Titel Jésus surnommé le Christ ${ }^{24}$, das in der Ewerbeckschen Sammlung vorliegt, schreibt er, dass "man bedauern mag, dass der Übersetzer weitere Schriften berücksichtigt hat, die bei weitem nicht denselben Wert besitzen«. Und er empfiehlt dagegen in diesem Band die Critique de l'histoire évangélique ${ }^{25}$ von Bruno Bauer: Man kann also umgekehrt daraus ableiten, dass die Werke von geringerem Wert, deren Abdruck er bedauert, jene von Daumer und Ghillany sind. ${ }^{26}$ Dennoch findet die Idee von der Konzession des hebräischen an die phönizischen Kulte ihren Weg und wird dabei vom Organ für die Verbreitung deutschen Denkens in Frankreich, der Revue germanique, übermittelt. Ein anderes Werk, das in dieser Zeitschrift von A. Nefftzer vorgestellt und übersetzt wird, geht zwar nicht so weit zu behaupten, dass der Kult von Moloch und Jehova seit den Anfängen gleich gewesen wären, doch äußert es die Hypothese, wonach die Molochriten zur Zeit der Könige bei den Hebräern einen so festen Platz hatten, dass die "frühen Übersetzer« darauf bedacht waren, deren Spuren zu verwischen und alle Mittel ihrer Kunst einsetzten, "um so weit wie möglich die Verwechslung zwischen Jehova und den fremden Elohim zu unterbinden «. ${ }^{27}$ Dies kommt Daumers These sehr nahe, wonach die heiligen Bücher alle nachträglich bearbeitet wurden, um die Ähnlichkeit zwischen hebräischem und phönizischem Kult zu verbergen. ${ }^{28} \mathrm{Kommen}$ wir zu Patrice Larroque zurück, der Daumers Thesen von vorneherein so ablehnend gegenüberstand. Wir wissen, dass Flaubert die Revue germanique liest, allerdings können wir nur vermuten, welche Artikel seine Aufmerksamkeit geweckt haben; dagegen sind wir gewiss, dass er sich mit den »antikatholischen Einwänden vollstopft", die in Examen critique des doctrines de la religion chrétienne enthalten sind. Wie könnte das Kapitel über "Sacrifices humains" (Menschenopfer) in der Bibel einem so eifrigen Leser entgangen sein, vor allem einem Leser, in dessen Schreibhorizont "la grillade des moutards" (Kinderopfer) liegt?

»So erlaubt Moses, der an anderen Stellen Menschenopfer untersagt, diese nicht nur, sondern schreibt sie sogar vor für den Fall, da man Gott ein solch unfrommes Gelübde abgelegt hat! Dies ist ein grauenvoller Widerspruch, dem man unmöglich entkommen kann, so sehr sind die einschlägigen Texte präzise. So kann man in Kapitel 11 der Richter sehen, dass Jeftah diese Vorschrift an seiner eigenen Tochter in die Tat umsetzt [...]. Man kann in alledem nur einen Überrest jenes verabscheuungswürdigen alten Kultes sehen, bei dem der Gottheit Menschenopfer dargebracht werden. Möge der Leser mit dem Kult der Phönizier und der Karthager vergleichen, diesem Molochkult, dem die Israeliten so zugeneigt waren, wie man es 
auf jeder Seite ihrer Geschichte sieht, den sie trotz Tadel und Drohungen ihrer Propheten immer weiter betrieben und erst in der Gefangenschaft den Gefallen daran verloren und ihn aufgaben. $\aleph^{29}$

»Möge der Leser mit dem Kult der Phönizier und der Karthager vergleichen«: Genau dazu gibt Salammbô Gelegenheit.

Bezüglich Jeftahs Opferung greift Larroque seinerseits die Kritik an jenen Übersetzungspraktiken auf, die den heidnischen Charakter des hebräischen Kults verbergen sollten:

"Mittels gewisser Eingriffe, die gleichermaßen übersetzerische Untreue sind, unterstreicht Abbé von Genoude, dass Jeftah seine Tochter nicht opferte, sondern sich damit begnügte, sie der Keuschheit zu weihen. Danach erklärt er, dass in seiner Übersetzung das Problem verschwindet, das die Ungläubigen mit diesem Menschenopfer haben, das dem Gott der Bibel dargebracht wird. Zweifelsohne durch seine Übersetzung. Leider bedeutet übersetzen, so wie er es tut, nicht mehr übersetzen, sondern Texte verändern. Die Bibel spricht entschieden und deutlich von einem Brandopfer, das Gott dargebracht wird, und jeder weiß, dass die Brandopfer des mosaischen Gesetzes tatsächliche, physische Opfer waren und dass, was dazu in den Büchern des Alten Testaments gesagt wird, im eigentlichen und nicht nur im übertragenen oder allegorischen Sinn zu verstehen ist. $\ll^{30}$

Larroque schließt sich damit sachlich Daumers und Feuerbachs Meinung an, und die von uns zitierten Auszüge aus Examen critique de la Bible zeigen, dass er unter dem Einfluss dieser Autoren steht, die er ansonsten ablehnt. Diese gelten als heimliche Referenz für das freie Denken im Frankreich jener Zeit: Sie werden kritisiert, zugleich jedoch plagiiert und bilden so den Hintergrund für die neue historische Bibel-Kritik.

11 Auf ihnen gründet dieser epistemologische Umschwung, diese Revolution, an der sich all jene beteiligen, die den modernen Kampf gegen die »Infame« führen: Die vorgeblich zivilisierte und zivilisatorische christliche Religion ${ }^{31}$ ist barbarisch. Hier wird sie auf den Kopf gestellt, wird ihre Wahrheit durch das Aufzeigen dessen sichtbar gemacht, was der gesunde Menschenverstand für ihr Gegenteil hält. „Gott ist der Dämon«, sagte Ewerbeck $^{32}$ mit Bezug auf die »göttliche Gerechtigkeit«:

"So ist die Gnade Gottes nur ein anderer Name für die Freiheit Gottes oder die Gerechtigkeit Gottes; der furchtbare Sinn dieser drei Worte bleibt immer derselbe: nämlich Laune, Willkür dieses absoluten, väterlichen Königs des Universums, der da sagt: Denn ich finde Gefallen daran. Gott ist der Dämon.«

Die Idee von einem Gott der Christen, die von einer unfrommen Konzeption des Göttlichen ausgeht, ist bei den kritischen Exegeten des Christentums in Deutschland und Frankreich weit verbreitet. Flaubert formuliert dies in einem Brief an Edma Roger des Genettes, in dem er sich für seine »antireligöse Wut« rechtfertigt, wie folgt:

"Sie sind über meine antireligiöse Wut erstaunt, und der unmittelbare Grund dafür ist folgender: in jedem Augenblick meiner Studien stoße ich auf die Bibel, und in der Bibel auf den gegenwärtigen Gott, auf den der Katholiken, der mich wegen seiner beschränkten, engstirnigen, orientalischen und monarchischen Seite immer mehr verzweifeln lässt. Das ist ein Ludwig XIV., ein Sultan, irgendetwas Menschenähnliches, das mir letztlich sehr kläglich erscheint und dessen Konzept mir sehr unheilig vorkommt.«

„Vous vous étonnez de ma rage antireligieuse, en voici la raison immédiate : c'est qu'à chaque moment dans mes études, je touche à la Bible, et dans la Bible, au Dieu actuel, à celui des catholiques, qui m'exaspère de plus en plus par son côté restreint, borné, oriental, monarchique. C'est un Louis XIV, un sultan, je ne sais quoi d'humain, qui me semble, en définitive, très piètre et dont la conception me paraît très impie. $^{33}$ 
Wenn der "gegenwärtige Gott» sündigt, dann aufgrund seiner "monarchischen Seite«, sagt Flaubert; Ewerbeck sagte aufgrund seines Charakters eines »absoluten Königs«: melech, das heißt moloch, bedeutet auf hebräisch »König«. Wenn der katholische Gott seine »orientalische Seite« zeigt, dann ist das der Moloch in ihm, dieser König aus dem Orient, dieser "König" in orientalischer Sprache, der erscheint und seine Herkunft aus dem Götzenkult enthüllt, den er vorgeblich bekämpfen will. Gott ist Moloch, denn er ist Anti-Natur, Antiphysis:

"Die Zeit und Welt, die auf diesen holden Götterdienst, auf diese edle, affirmative Entwicklung des menschlichen Wesens [der griechischen Antike] folgte, war die der Einsiedler, Säulenheiligen, Mönche und Pfaffen, der die Natur hassenden, die menschliche Gesellschaft fliehenden, sich selbst mißhandelnden, alle Welt entzweienden, Mord und Tod predigenden Asceten und Fanatiker, die Zeit der Bußen und Peinigungen des Fleisches, der Glaubensinquisitionen, Schafote, Scheiterhaufen, Bartholomäusnächte, Hexenprozesse, Judenschlachten u.s.w., eine Zeit und Welt, deren Finsterniß, Unglück und Gräulichkeit [...] beispiel- und namenlos ist. ( $^{34}$

In Le Prêtre, la femme et la famille sagt auch Michelet: »[U]nsere Terroristen des 15. und 16. Jahrhunderts waren Mönche. Ein systematisch negatives Leben, ein Leben zum Tode entwickelt im Menschen lebensfeindliche Instinkte; wer leidet lässt gerne leiden. Die harmonischen und fruchtbaren Seiten unserer Natur, die mit Güte auf der einen, mit Genie, Erfindungsreichtum auf der anderen Seite zusammenhängen, halten diesem partiellen Selbstmord nicht stand. $\aleph^{35}$ Nach Erhalt des Buches schreibt Flaubert umgehend an Michelet, um ihm für die Zusendung zu danken, und dies mit so glühenden Worten, dass es mehr als bewundernde Höflichkeit ist, die man ansonsten von ihm kennt. Hier geht es um völlige Übereinstimmung:

"Als ich hier ankam, mein lieber Meister, habe ich mich auf Ihren Band gestürzt, und ich schreibe Ihnen eilig, überwältigt und geblendet von einer ersten Lektüre. [...] Sie sagen zugleich, was war und was ist (und was vielleicht leider! noch lange sein wird); Sie haben einen ewigen Priester geschaffen [...]. Der große Voltaire beendete selbst seine geringsten Botschaften mit `Ecr. l'inf‘ [Zerstört die Infame]. Ich habe keineswegs die Autorität, diese Worte zu wiederholen. Jede Ermutigung meinerseits an Sie wäre lächerlich, doch ich drücke Ihnen beide Hände im Hass auf die Anti-physis."

»En arrivant ici, mon cher Maître, je me suis précipité sur votre volume, et je vous écris à la hâte, dans l'émotion, l'éblouissement d'une première lecture [...]. Vous dites à la fois ce qui a été et ce qui est (et peut-être, hélas ! ce qui sera encore pendant longtemps); vous avez fait un prêtre éternel [...]. Le grand Voltaire finissait ses moindres billets par : $/ E c r$. l'inf. J Je n'ai aucune autorité pour redire cette parole. De moi à vous, tout encouragement serait ridicule, mais je vous serre les mains dans la haine de l'anti-physis. «16

Hier wird mit der Präterition der Voltaireschen Briefunterschrift eine Kette des Kampfes gegen die Infame in Gang gesetzt, die unter der Schirmherrschaft dieses wahren Heiligen steht. Der »ewige Priester", Todbringer, Töter der Natur, das ist der katholische Priester; aber Flaubert, der sich in der Schlussphase seiner Arbeit an Salammbô befindet, denkt sicher auch an Schahabarim, seinen Priester aus Karthago. Dieser wird übrigens später in dem berühmten Brief vom Dezember 1862 an SainteBeuve zu den »braven Männern von Port-Royal« in Parallele gesetzt:

»Eine letzte Frage, Meister, eine unziemliche Frage: Warum finden Sie Schahabarim fast komisch, Ihre braven Männer von Port-Royal aber so ernsthaft? [...] Denn ich glaube an Port Royal, und ich wünsche noch weniger dort zu leben als in Karthago. Auch das war exklusiv, widernatürlich, gezwungen, ganz aus einem Stück und doch wahr.» 
"Une dernière question, ô Maître, une question inconvenante: pourquoi trouvezvous Schahabarim presque comique et vos bonshommes de Port-Royal si sérieux! [...] j'y crois, à Port-Royal, et je souhaite encore moins y vivre qu'à Carthage. Cela aussi était exclusif, hors nature, forcé, tout d'un morceau, et cependant vrai.« ${ }^{37}$

Das Gemeinsame an Michelets Priestern, Sainte-Beuves braven Männern und dem Priester aus Salammbô ist ihr "widernatürlicher" Charakter. Es ist auch der Wahn. Schon 1859 wird Salammbô als eine »Geistesgestörte« entworfen, die Flaubert in einem Brief an Mademoiselle Leroyer de Chantepie implizit vergleicht mit »diesem jungen Mädchen, Ihre Verwandte, die infolge religiöser Ideen verrückt wurde«. Und er fügt hinzu:

»aber das ist eine bekannte Geschichte. Man muss ein robustes Temperament haben, um auf die Gipfel des Mystizismus zu steigen, ohne den Kopf zu verlieren [...] Ich bin in letzter Zeit nebenbei $\mathrm{zu}$ jenen psychisch-medizinischen Studien zurückgekehrt, die mich vor zehn Jahren, als ich meinen Saint Antoine schrieb, so sehr in Bann geschlagen haben. Bezüglich meiner Salammbô habe ich mich mit Hysterie und geistiger Verwirrung befasst.«

"mais c'est une histoire commune. Il faut avoir le tempérament robuste pour monter sur les cimes du mysticisme sans y perdre la tête [...]. Moi, dans ces derniers temps, je suis revenu incidemment à ces études psycho-rnédicales qui m'avaient tant charmé il y a dix ans, lorsque j'écrivais mon Saint Antoine. À propos de ma Salammbô, je mes suis occupé d'hystérie et d'aliénation mentale. ${ }^{38}$

Der Zusammenhang zwischen Wahn und Religion ist eine ständige Obsession Flauberts, worauf hier der Bezug auf Saint Antoine hinweist. Doch hat er nicht nur mit seiner persönlichen Geschichte zu tun. Man muss ihn auch innerhalb der antichristlichen Argumentationen seiner Zeit sehen; jener von Michelet ${ }^{39}$ ebenso wie von Daumer:

»Das Christentum ist verantwortlich für die religiösen psychischen Störungen, von denen Europa im Vorfeld des Jahres 1000 heimgesucht wurde; es ist verantwortlich für die religiösen psychischen Störungen, die unter den Bezeichnungen von Schüttelkrämpfen, Flagellantentum, Sankt-Michaels-Wallfahrten, Totentanz, Veitstanz [Chorea, A.d.Ü.] und vor allem des Mönchtums bekannt sind.«40

Wenn Flaubert 1860 feststellt, dass jener Wahn, den er seine »antireligiöse Wut« nennt, wieder da ist, besteht kein Zweifel daran, dass dies mit Salammbô zusammenhängt. Der Roman war Schauplatz für eine unterschwellige theologische Reflexion, die den heidnischen Gott auf den jüdisch-christlichen Gott reagieren ließ: Während der Romancier aus dokumentarischen Gründen und auch, weil die Quellen zu Karthago im Vergleich zum immensen Kontinent der Bibel mager waren, den Moloch in Jehova suchte, fand er Jehova in Moloch. Ob dieser Fund Zufall war oder Absicht, ob der Romancier der These, auf die er stößt, beipflichtet oder nicht, werden wir nicht erfahren. Man kann sich allerdings kaum vorstellen, dass Flaubert mit dem Wissen, das er hat, nicht weiß, was er tut, wenn er die Statue des verschlingenden Moloch erneut in Gang setzt. Und wenn das Gastmahl, zu dem die Leser geladen sind, an dem sie objektiv teilhaben, nicht die Rekonstruktion eines absonderlichen, verflossenen Kultes wäre, sondern das Wiederaufleben eines Ritus, der mit dem Ursprung des Christentums selbst verknüpft ist, der es erklärt und ganz in sich enthält; also nicht eine fremde, sondern verfremdete Praxis wäre, die Kommunion zurückgeführt auf ihr anthropophagisches Wesen; nicht Schauspiel eines bildgewaltigen Heidnischen, sondern Darstellung des Heidentums innerhalb des Christentums, also des Christentums als Heidentum? In diesem Fall müsste man sagen: Salammbô - das sind wir. 


\section{BIBLIOGRAPHIE}

Bauer, B. (1841/42): Kritik der evangelischen Geschichte der Synoptiker, 3 Bde., Leipzig: Otto Wiegand.

Daumer, G. F. (1842): Der Feuer- und Molochdienst der alten Hebräer, Braunschweig: Fr. Otto. Im Internet: http://books.google.de/books?

id=qnFAAAAAcAAJ\&printsec=frontcover\&hl=de\&source=gbs_ge_summary_r\&cad=0\#v=onepage\&q\&f=false

Daumer, G. F. (1847): Die Geheimnisse des christlichen Alterthums, 2 Bde., Hamburg: Hoffmann und Campe. Im Internet, Bd. I: http://books.google.de/books?

$\mathrm{id}=\mathrm{lbAAAAAAcAAJ} \&$ printsec $=$ frontcover\&hl=de\&source=gbs_ge_summary_r\&cad $=0 \# \mathrm{v}=$ onepage\&q=Chorea\&f=false

Ewerbeck, H. (1850a): Qu'est-ce que la Bible, d'après la nouvelle philosophie allemande, [enthält: „Le Culte du Moloch chez les Hébreux de l'antiquité« (= Daumer [1842]) und „Secrets de l'antiquité chrétienne«, von Daumer (= Daumer [1847]); »Les Sacrifices humains chez les Hébreux de l'antiquité«, von Ghillany (= Ghillany [1842]); »Jésus surnommé le Christ«, von Lützelberger (= Lützelberger [1842]); »Critique de l'histoire évangélique des synoptiques«, von Bruno Bauer (= Bauer [1841/42])], Paris: Ladrange.

Ewerbeck, H. (1850b): Qu'est-ce que la religion, d'après la nouvelle philosophie allemande, Paris: Ladrange.

Feuerbach, L. (2006): Das Wesen des Christentums, 3. durchges. Aufl. Berlin: Akademie-Verlag.

Flaubert, G. (1971): Salammbô, in: Euvres complètes, Bd. II, Paris: Club de l'Honnête Homme.

Flaubert, G. (1977): Briefe, hg. u. übers. von H. Scheffel, Stuttgart: Goverts.

Flaubert, G. (1979): Salammbô, hg. von M. Bosse u. A. Stoll, übers. von G. Brustgi, Frankfurt/M.: Insel.

Flaubert, G. (1980): Correspondance, Bd. 2, Paris: Gallimard (Pléiade).

Flaubert, G. (1991): Correspondance, Bd. 3, Paris: Gallimard (Pléiade).

Flaubert, G. (2001): Salammbô, hg. von G. Séginger, Paris: Garnier-Flammarion.

Geiger, A. (1857): Urschrift und Übersetzungen der Bibel in ihrer Abhängigkeit von der inneren Entwicklung des Judenthums, Breslau: Julius Hainauer.

Ghillany, F. W. (1842): Die Menschenopfer der alten Hebräer, Nürnberg: Johann Leonhard Schrag. Goncourt, E. u. J. (1986 [1869]): »Madame Gervaisais«, CEuvres complètes, XXXI, Genf, Slatkine reprints.

Larroque, P. (1860): Examen critique des doctrines de la religion chrétienne, 2 Bde., Paris: Bohné et Schultz.

Lützelberger, E. C. J. (1842): Jesus, was er war und wollte, und wie er zum Christus wird, Nürnberg: Julius März. Im Internet: http://books.google.de/books? $\mathrm{id}=\mathrm{dXdAAAAAcAAJ} \&$ printsec $=$ frontcover\&hl=de\&source=gbs_ge_summary_r\&cad=0\#v=onepage \&q\&f=false Michelet, J. (1861 [1845]): Le Prêtre, la femme et la famille, neue Ausgabe, Paris: Chamerot.

Mondon, G. (2002): Genèse du personnage de Salammbô d'après les manuscrits autographes de Gustave Flaubert, Doktorarbeit der Universität Paris IV, verteidigt am 25. Januar 2002 unter dem Vorsitz von Pierre-Marc de Biasi. 
Neffzer, A. (1860): »La Bible et les partis chez les anciens isréalites«, Revue germanique, Bd. IX, Januar-März, S. 96-124.

Poliakov, L. (1987): Geschichte des Antisemitismus, Bd. 6: Emanzipation und Rassenwahn, übers. von R. Pfisterer, Worms: Georg Heintz.

Renan, E. (1850): »Qu'est-ce que la religion dans la nouvelle philosophie allemande?«, La liberté de penser, Bd. VI, Juni-November.

Renan, E. (1855): Histoire générale et système comparé des langues sémitiques, Paris: Imprimerie nationale.

Renan, E. (1859): Nouvelles considérations sur le caractère général des peuples sémitiques, et en particulier sur leur tendance au monothéisme, Paris: Impr. Impér.

\section{NOTES}

1. "Sie wundern sich über meine antireligiöse Wut«, schreibt Flaubert an Edma Roger des Genettes in einem Brief, den Jean Bruneau auf 1860 datiert. Vgl. Flaubert (1991), S. 131: „Vous vous étonnez de ma rage antireligieuse .

2. Brief von Frédéric Baudry an Flaubert, 27. Oktober 1859: »Mein lieber Freund, Ich lese gerade Renans Nouvelles considérations sur le caractère général des peuples sémitiques, et en particulier sur leur tendance au monothéisme (Neue Betrachtungen über den allgemeinen Charakter der semitischen Völker und insbesondere über ihren Hang zum Monotheismus), Paris, Impr. Impér., 1859, broch. In 8, Auszug aus Nummer 3 des Jahrgangs 1859 des Journal Asiatique, und finde darin einen Abschnitt, der Sie bezüglich des Peplos der Astarté interessieren dürfte [...] Ich habe Renan angekündigt, dass ich Sie einander vorstellen werde. Er erwartet Sie mit Ungeduld und Neugierde« (New York, Pierpont Morgan Library, Heineman Collection, MS 83, "Carthage, notes diverses«, 74 S., fo 75, 76). Tatsächlich trafen sich beide Männer in ihrem Interesse für Karthago im Zusammenhang mit dem Zaimph [Heiligen Schleier].

3. Zum »Polygenismus«, den Maury verteidigt, vgl. beispielsweise Athenaeum français, S. 10981100, Nr. 47, 19. November 1853.

4. Flaubert (1991), S. 82 (Paris, Mittwoch, 28. März 1860, an Louis Bouilhet): „Ce même dimanche il y avait eu chez moi un grand combat entre Baudry, Saint-Victor et l'excellent père Maury, qui est charmant. Je dîne demain à Versailles avec lui et Renan.«

5. Flaubert (1991), S. 91: "Je recalle enfin mon interminable chapitre VII qui ne sera pas venu sans peine. De plus je prépare le huitième. Je me bourre des objections anticatholiques du père Larroque, je lis le dernier volume de Michelet et divers articles d'exégèse dans 1a Revue germanique. Depuis mon retour j'ai écrit 15 pages, ce qui est bien joli pour un empêtré comme moi.«

6. La Bible, Neuübersetzung mit Gegenüberstellung des hebräischen Textes mit philologischen, geografischen und literarischen Anmerkungen, Paris, 1831-1851 (18 Bände). Flauberts Anmerkungen zur Bibel von Cahen sind in der Pierpont Morgan Library in New York erhalten unter der Signatur MS 88. Sie bestehen aus 37 Folioblättern, nummeriert von 305 bis 342 .

7. Aus dem zitierten Brief, vgl. Fußnote 2.

8. Renan (1855), S. $171 \mathrm{f}$.

9. Renan (1855), S. $178 \mathrm{f}$.

10. Renan (1855), S. $186 \mathrm{f}$.

11. Renan (1859). Auszug aus Nummer 3 des Jahrgangs 1859 des Journal Asiatique, S. 65.

12. Das Dossier umfasst 21 handgeschriebene Folioblätter, die in der Bibliothèque Nationale de France unter der Signatur NAF 23662 aufbewahrt sind. Der Titel, um den es hier geht, erscheint 
in fo 158. Das Dossier wurde in die Ausgabe der Cuvres complètes von Flaubert, Club de l'Honnête Homme, Bd. II, 1971, S. 489-512, aufgenommen.

13. Ewerbeck (1850a). Ein weiterer Band (1850b), im gleichen Jahr beim gleichen Verlag erschienen, besteht ausschließlich aus Feuerbach-Texten, die von Ewerbeck übersetzt wurden.

14. "Die Gefahr drängt, denn hier wie dort ist der Feind des Menschengeschlechts in Form des Wiedergängers des Herrn Grafen Joseph de Maistre wieder aufgetaucht«, S. VI des Vorworts (Ewerbeck [1850a]).

15. Ewerbeck (1850a), S. 1-48. [Übersetzt nach der französischen Vorlage; das deutsche Original (Daumer [1842] weist keine Thesenform auf; A.d.Ü.]

16. Mondon(2002).

17. Dies trifft nicht auf seinen Schüler Ghillany zu, der im gleichen Band eindeutig antisemitische Gedanken entwickelt. Zur Frage des Antisemitismus der revolutionären deutschen Philosophen und der unheilvollen Zukunft der These vom »jüdischen Molochismus« vgl. die Synthese von Poliakov (1987), im Kapitel »Der Kreuzzug der Atheisten«, S. 216-236.

18. Ewerbeck (1850a), S. 54-57 [Daumer (1847], S. 5].

19. Ewerbeck (1850b), S. 64-498: "L’Essence du christianisme. Louis Feuerbach (1842)«, S. 73: "Eine historisch-philosophische Analyse, im Unterschiede von den nur historischen Analysen des Christentums. Der Historiker zeigt, z. B. wie Daumer und Ghillany, daß das Abendmahl ein aus dem alten Menschenopferkultus stammender Ritus ist, daß einst statt des Weines und Brotes wirkliches Menschenfleisch und -blut genossen wurde.« [zitiert nach dem deutschen Original: Feuerbach (2006), S. 22; A.d.Ü.]

20. Ewerbeck (1850b), S. 427 und S. 446.

21. Ewerbeck (1850b), S. 446.

22. Flaubert (1979), S. 307; frz.: Flaubert (2001), S. 267: „Seigneur! mange!«

23. Renan (1850).

24. [Lützelberger (1842); A.d.Ü.]

25. [Bauer (1841/42); A.d.Ü.]

26. Larroque (1860), S. 246. Vgl. in Flaubert (1991), S. 137, den Brief an Mademoiselle Leroyer de Chantepie vom 15. Januar 1861: "Haben Sie das Examen des dogmes de la religion chrétienne von P. Larroque? Es gehört zu Ihren Lieblingslektüren. Der Autor ist bis $\mathrm{zu}$ den Quellen zurückgegangen, eine seltene Sache! und ich sehe keinen ernstzunehmenden Einwand, den man ihm entgegenhalten könnte. Es ist eine umfassende Widerlegung des katholischen Dogmas.«"Avez-vous l'Examen des dogmes de la religion chrétienne, par P. Larroque? Cela rentre dans vos lectures favorites. L'auteur est remonté aux sources, chose rare! et je ne vois pas une objection sérieuse qu'on puisse lui poser. C'est une réfutation complète du dogme catholique.«

27. Neffzer (1860), S. 96-124, Zusammenfassung und Übersetzung von Geiger (1857), hier: S. 118119.

28. Nach dem Tod von König Salomo stürzt ein reformerischer »neuer Jehovismus« schließlich die Idole »und man macht sich daran, die historischen Bücher der Nation zu überarbeiten [...] Wir finden, dass der nationale Reformwille nach Salomo die Berichte über die Grausamkeiten der Vorfahren sorgfältig ausgelöscht oder zumindest verschleiert hat, ohne bei diesem Vorhaben jedoch mit vollständiger Sorgfalt vorzugehen«, Ewerbeck (1850a), S. 3 [Vgl. Daumer (1842), S. 3, Fußnote 1: »[...] die gräßlichsten Kinderopfer waren bei den Hebräern bis in's Exil hinein im Gange, und diese waren kein Abfall von Altnationalem zu Fremden und keine Neuerung, sondern im Gegentheil eine Fortsetzung des Altnationalen, ein Hängen an demselben, im Gegensatze gegen den reformirten Jehovismus, der auch die hebräische Sagengeschichte in den biblischen Darstellungen in sein Licht stellte und das Neue als ein Alterthümliches erscheinen ließ.« A.d.Ü.]

29. Larroque (1860), Bd. II, S. $44 \mathrm{f}$.

30. Larroque (1860) Bd. II, S. 98. 
31. Dies ist ein Topos der Kolonialherren und Missionare des 19. Jahrhunderts, der beispielsweise in Madame Gervaisais der Gebrüder Goncourt bekämpft wurde, einem Roman, den Flaubert bei dessen Erscheinen 1869 begrüßte. Die Heldin, eine Pariser »Philosophin«, die sich in Rom niedergelassen hat, ist angewidert vom barbarischen Schauspiel einer Prozession. Sie schreibt an ihren Bruder: "Ich sagte mir: dies ist immerhin der Glaube der Zivilisation; und ich sah nichts als einen wilden und gänzlich rohen Götzendienst des Orients, ein bisschen wie die Prozession der Inder zu Ehren des Jagannath-Idols!« Goncourt (1986), S. 49.

32. Ewerbeck (1850b), S. 321.

33. Flaubert (1991), S. 321.

34. Daumer (1847), S. 7 f.

35. Michelet (1861), S. 334.

36. Flaubert (1991), S. $155 \mathrm{f}$.

37. Flaubert (1977), S. 458 f.

38. Flaubert (1991), S. 16 f.

39. Michelet (1861), S. 237: "Ich bitte Sie, wo liegt die Grenze, die unsere heutigen Klöster von den Zwangsanstalten und den Häusern trennt, in denen man Verrückte einsperrt?«

40. Ewerbeck (1850a), S. 104. [Vgl. Daumer (1847); A.d.Ü.]

\section{INDEX}

Schlüsselwörter : antireligiöse Wut, Antiklerikalismus, Christentum, Monotheismus, Moloch, Jehova, Wahn

Mots-clés : rage antireligieuse, anticléricalisme, christianisme, monothéisme, Moloch, Jéhovah, folie

\section{AUTEURS}

\section{AGNÈS BOUVIER}

Agnès Bouvier ist Post-doc im Forschungslabor Institut des textes et manuscrits modernes (ITEM). 